$$
\text { CONF- } \begin{gathered}
95 / 0263--14 \\
\text { UCRL-JC-122314 } \\
\text { PREPRINT }
\end{gathered}
$$

\title{
HEAVY ION FUSION EXPERIMENTS AT LLNL
}

\author{
J.J. Barnard, M.D. Cable, D.A. Callahan, F.J. Deadrick, S. Eylon, ${ }^{\dagger}$
}

T.J. Fessenden," A. Friedman, D.P. Grote, K.A. Holm, H.A. Hopkins,

D.L. Judd, ${ }^{+1}$ R.L. Hanks, S.A. Hawkins, H.C. Kirbie, B.G. Logan,

S.M. Lund, L.A. Nattrass, D.B. Longinotti, ${ }^{+++}$M.B. Nelson,

M.A. Newton, C.W. Ollis, T.C. Sangster, and W.M. Sharp

Lawrence Livermore National Laboratory, University of California

P.O. Box 5508, L-440, Livermore California 94550, USA

${ }^{\dagger}$ Participating Guest at

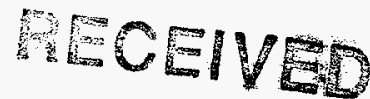

Lawrence Livermore National Laboratory, University of California

P.O. Box 5508, L-440, Livermore California 94550, USA

${ }^{+t}$ Lawrence Berkeley Laboratory, University of California
Berkeley California 94720, USA JUN 051328

OSTI

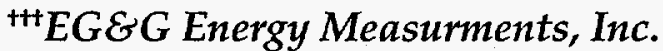 \\ Pleasanton, California 94588, USA
}

This paper was prepared for submittal to the

Proceedings of the 8th ICFA Advanced Beam Dynamics Workshop on Space Charge Dominated

Beams and Applications of High Brightness Beams

Bloomington, IN

October 10-13, 1995

February 6, 1996

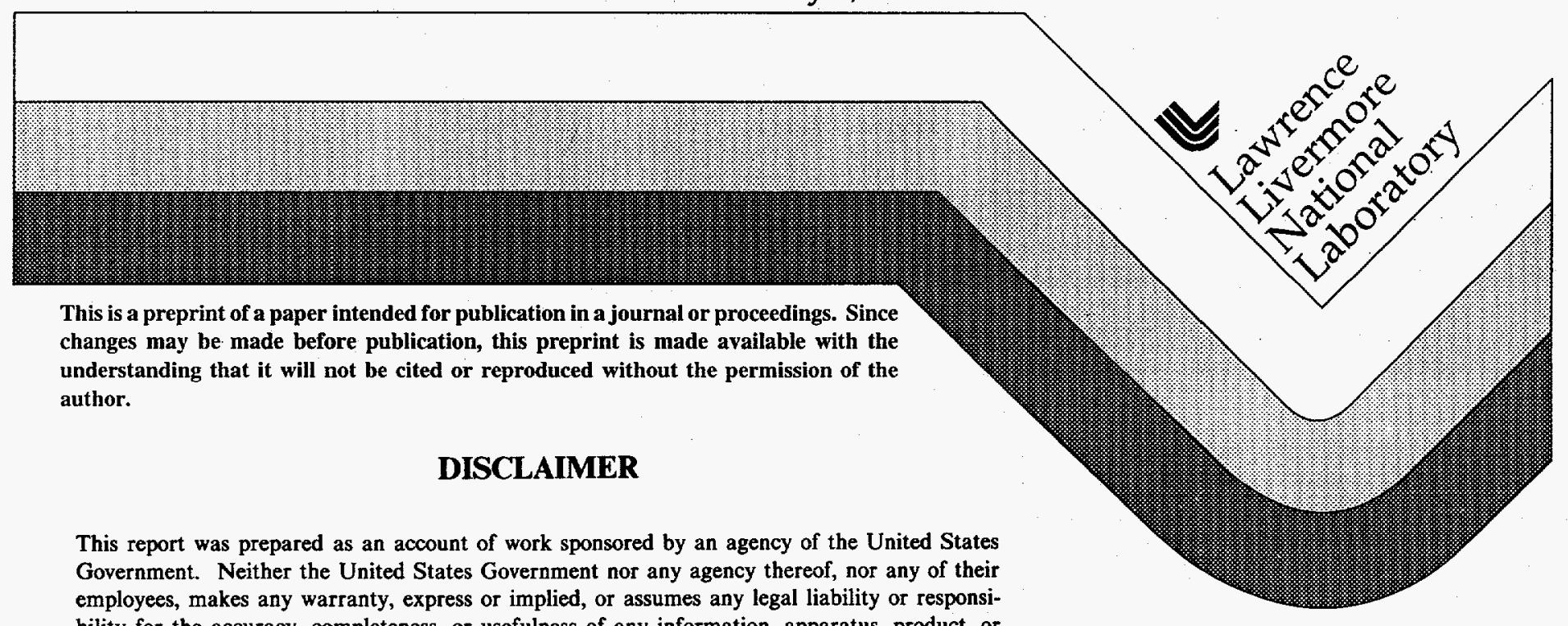
bility for the accuracy, completeness, or usefulness of any information, apparatus, product, or process disclosed, or represents that its use would not infringe privately owned rights. Reference herein to any specific commercial product, process, or service by trade name, trademark, manufacturer, or otherwise does not necessarily constitute or imply its endorsement, recommendation, or favoring by the United States Government or any agency thereof. The views and opinions of authors expressed herein do not necessarily state or reflect those of the United States Government or any agency thereof. 


\section{DISCLAIMER}

This document was prepared as an account of worksponsored by an agency of the United States Government. Neither the United States Government nor the University of California nor any of their employees, makes any warranty, express or implied, or assumes any legal liability of responsibility for the accuracy, completeness, or usefulness of any information, apparatus, product, or process disclosed, or represents that its use would not infringe privately owned rights. Reference herein to any specific commercial products, process, or service by trade name, trademark, manufacturer, or otherwise, does not necessarily constitute or imply its endorsement, recommendation, or favoring by the United States Government or the University of California. The views and opinions of authors expressed herein do not necessarily state or reflect those of the United States Government or the University of California, and shall not be used for advertising or product endorsement purposes. 


\section{DISCLAMMER}

Portions of this document may be illegible in electronic image products. Images are produced from the best available original document. 
Eighth ICFA Advanced Beam Dynamics Workshop on Space Charge Dominated Beams and Applications of High Brightness Beams, Bloomington, Indiana, October 10-13, 1995

\title{
HEAVY ION FUSION EXPERIMENTS AT LLNL
}

J.J. Barnard ${ }^{1}$, M.D. Cable ${ }^{1}$, D. A. Callahan ${ }^{1}$, F. J. Deadrick ${ }^{1}$, S. Eylon $^{4}$, T.J. Fessenden ${ }^{2}$,A. Friedman ${ }^{1}$, D.P. Grote ${ }^{1}$, K.A. Holm ${ }^{1}$, H.A. Hopkins ${ }^{1}$, D.L. Judd ${ }^{2}$, R. L. Hanks ${ }^{1}$, S.A. Hawkins ${ }^{1}$, H.C. Kirbie ${ }^{1}$, B.G. Logan ${ }^{1}$, S.M. Lund ${ }^{1}$, L.A. Nattrass ${ }^{1}$, D. Longinotti ${ }^{3}$, M. B. Nelson ${ }^{1}$, M.A. Newton ${ }^{1}$, C.W. Ollis ${ }^{1}$, T.C. Sangster ${ }^{1}$, and W.M. Sharp ${ }^{1}$

${ }^{1}$ Lawrence Livermore National Laboratory ${ }^{2}$ Lawrence Berkeley National Laboratory ${ }^{3} \mathrm{EG} \& \mathrm{G},{ }^{4}$ Participating Guest at LLNL

\begin{abstract}
We review the status of the experimental campaign being carried out at Lawrence Livermore National Laboratory, involving scaled investigations of the acceleration and transport of space-charge dominated heavy ion beams. The ultimate goal of these experiments is to help lay the groundwork for a larger scale ion driven inertial fusion reactor, the purpose of which is to produce inexpensive and clean electric power.
\end{abstract}

\section{Introduction}

Because of their high efficiency, high repetition rate, and relatively simple target chamber geometries, heavy-ion accelerators are attractive candidates as drivers for inertial fusion energy power plants. Recirculating induction accelerators have been studied ${ }^{1,2}$ as potential lower cost alternatives to linear induction machines. The cost advantage is achieved, as in any circular accelerator, by the reuse of the accelerating and focusing components. However, the introduction of bends and the higher repetition frequency of the induction core pulsers introduces new challenges, as will be discussed below. A series of experiments leading to the development of a small ( $2 \mathrm{~m}$ radius) scaled recirculator at LLNL is underway. The ion source, matching section and a short linear transport section have been constructed and the beam has been characterized. A design for the half-lattice periods of the ring has been developed as well. A complete description of the experimental apparatus and results through October, 1995 have recently been presented in the literature ${ }^{3}$. The purpose of this paper is to summarize these results as well as present subsequent developments.

The ultimate specifications for a heavy ion driver are determined by the power and energy density requirements of the target, and the associated high beam quality requirements needed to ensure that the beam can be focused onto a small spot at the target, necessary for high energy density. Typical requirements ${ }^{4}$ set by target include a pulse energy of $\sim 4 \mathrm{MJ}$, a main pulse duration of $\sim 10 \mathrm{~ns}$, and an ion range in the radiation converter material of $\sim 0.1-0.2 \mathrm{~g} / \mathrm{cm}^{2}$. For the heaviest ion mass of about $200 \mathrm{amu}$, the total current is minimized (at $\sim 40 \mathrm{kA}$ ), and the individual ion energy required is $\sim 10 \mathrm{GeV}$. The required beam spot radius is in the range $\sim 1.5-3 \mathrm{~mm}$. Achieving this small spot, assuming unneutralized ballistic propagation through a final set of quadrupole lenses, requires that the normalized transverse emittance be 
less than $\sim 10 \mathrm{~mm}-\mathrm{mrad}$, and the longitudinal fractional momentum spread $\delta p / p$ be less than $\sim 1 \times 10^{-3}$. In contrast, the planned recirculator experiments will have a final current $(\sim 8 \mathrm{~mA})$, energy $(\sim 320 \mathrm{keV})$, and normalized emittance ( $\sim 0.2 \mathrm{~mm}$-mrad) all much lower than the driver. Nevertheless, the relevant dimensionless parameters describing beam propagation and physics, (for example, undepressed phase advance $\sigma_{0}$, depressed phase advance $\sigma$, perveance $K$, and tune $\nu$ ) are in regimes which are either comparable to or more demanding than in the driver recirculator ${ }^{6}$.

\section{Recirculator Plans}

The goal of the small recirculator experiment is to test nearly all of the beam dynamics issues and many of the technology issues facing a driver. When successful, the experiment will have demonstrated:

- transport of a space charge dominated beam around multiple laps of a circular accelerator,

- longitudinal and transverse beam control in a recirculator,

- emittance control (less than a factor of 2 growth is the goal),

- freedom from resonance instabilities,

- injection and extraction into and out of a ring,

- energy gain (by a factor of 4),

- bunch length compression (by a factor of 2).

The new technology demonstrated will include:

- high repetition rate pulse power with variable pulse format, including acceleration and longitudinal confinement fields,

- coordination of the temporally increasing dipole field with the variable firing of the induction cores, as the beam speed increases,

- beam steering over a muliple laps.

The experiment will not examine:

- stability of background gas against beam desorption,

- high current instabilities,

- manipulations of multiple beams.

The physics design of the small recirculator required a number of parametric choices that are more completely motivated elsewhere ${ }^{5,6}$. Simplicity, low cost, and relevance to a driver-scale machine provided general guidance in the design. The dipoles are ramped electric rather than ramped magnetic for simplicity and cost. The quadrupoles will be permanent magnets, simulating the driver designs which use superconducting magnets (which are also fixed in strength). The insertion/extraction section consists of a permanent magnet elliptical quadrupole, with electric dipoles serving the dual purposes of kickers on insertion and extraction, as well as bending the beam on normal laps. The diagnostics for the recirculator must be non-invasive. Capacitive probes have been selected for determining the location of the beam centroid, providing sufficient information to steer the beam. The vacuum must be $<10^{-8}$ torr, so metallic seals are being used to minimize outgassing. This is still a large residual gas density compared to a driver design ${ }^{1}$, which requires pressures less than $\sim 10^{-10}$ torr. The maximum repetition rate of the induction core pulsers is $\sim 100 \mathrm{kHz}$, equal to the lap time around the recirculator. A prototype pulser has been developed which uses solid state switches to achieve the variable repetition rate and pulse format required for recirculator operation ${ }^{10}$. A list of some of the design parameters of the recirculator 
is given in table 1 , and a diagram illustrating the layout of the experiment is given elsewhere in these proceedings ${ }^{7}$.

\section{Linear Magnetic Transport Experiment}

Presently, our experiments are employing a straight magnetic transport section in preparation for bent beam studies. The apparatus consists of an injector, matching section, and magnetic transport section. The $80 \mathrm{keV}$ diode injector has a hot plate source which produces singly charged potassium ions. The injector is followed by seven electric quadrupoles which act as a matching section for converting the circular beam emerging from the diode into the elliptical shape needed for alternating gradient transport. This matching section was adapted from one of the transport sections of the LBNL Single Beam Transport Experiment ${ }^{9}$. Two of the original quadrupoles have been electrically separated so that dipole voltages may be applied for small steering corrections to the beam centroid. The matching section is followed by a drift section consisting of seven permanent magnet quadrupoles, each with pole tip fields of approximately $0.3 \mathrm{~T}$ (at a clear aperture radius of $3.5 \mathrm{~cm})$ and an integrated field gradient $\left(=\int B^{\prime} d z\right)$ of $0.95 \mathrm{~T}$, where $B^{\prime}$ is the quadrupole gradient and the integral is carried out along the quadrupole magnetic axis. Results to date are encouraging. No anomalies have been observed in magnetic transport of a space-charge-dominated beam. The goals of these experiments are to characterize the beam prior to injection into the ring, to develop necessary diagnostics for recirculator operation, and to assess the role that stray electrons play in beam transport. Some examples of data obtained from our initial experiments are briefly summarized below.

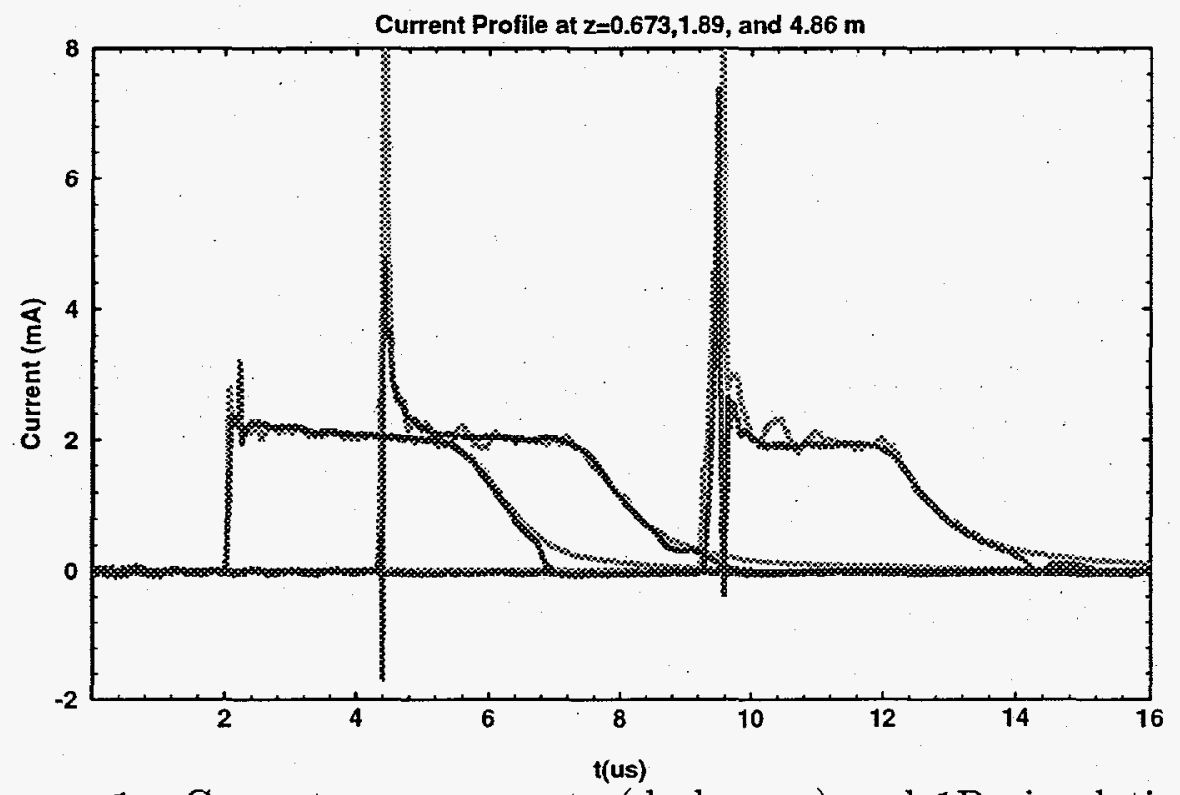

Figure 1. Current measurements (dark gray) and 1D simulations (light gray) at Faraday Cups located $0.67,1.89$, and $4.86 \mathrm{~m}$ downstream of the diode source.

Faraday cup current profiles: Figure 1 shows the time dependent current profile at three stages within the matching and transport section. 
The $2 \mathrm{~mA}$ flattop current is proceeded by a sharp current spike in the beam head. The spike arises because the rise time of the injector pulser is longer than optimal. Ions emitted at the beginning of the pulse are overtaken by faster, higher energy ions emitted slightly later. A new, faster pulser is in the process of being installed in the machine. The shorter rise time of this new pulser will produce a waveform closer to the ideal risetime ${ }^{11}$, so that the particles emitted during the rise will get a boost from the space charge field of the particles emitted later, so as to arrive at the diode exit with an energy and current nearly equal to those of the main body of the pulse. The new pulser should eliminate problems associated with the non-ideal waveform. Nevertheless the good agreement between simulations and experiment with the non-ideal pulse has given us confidence in the modeling effort and has served to enhance our understanding of the machine.

Energy analyzer data: An electrostatic energy analyzer in which the beam is bent by a transverse electric field which segregates particles based on kinetic energy, has been used as an independent energy check. Results from this diagnostic located $1.85 \mathrm{~m}$ from the source are shown in figure 2 . The energy flatness is about $\pm 3 \%$ over $3 \mu \mathrm{s}$. 1D code comparisons using the inferred injector waveform as input to the code agree with the absolute measure of the energy within $2 \%$ (essentially using time of flight as the energy measure), and show virtually identical energy flatness.

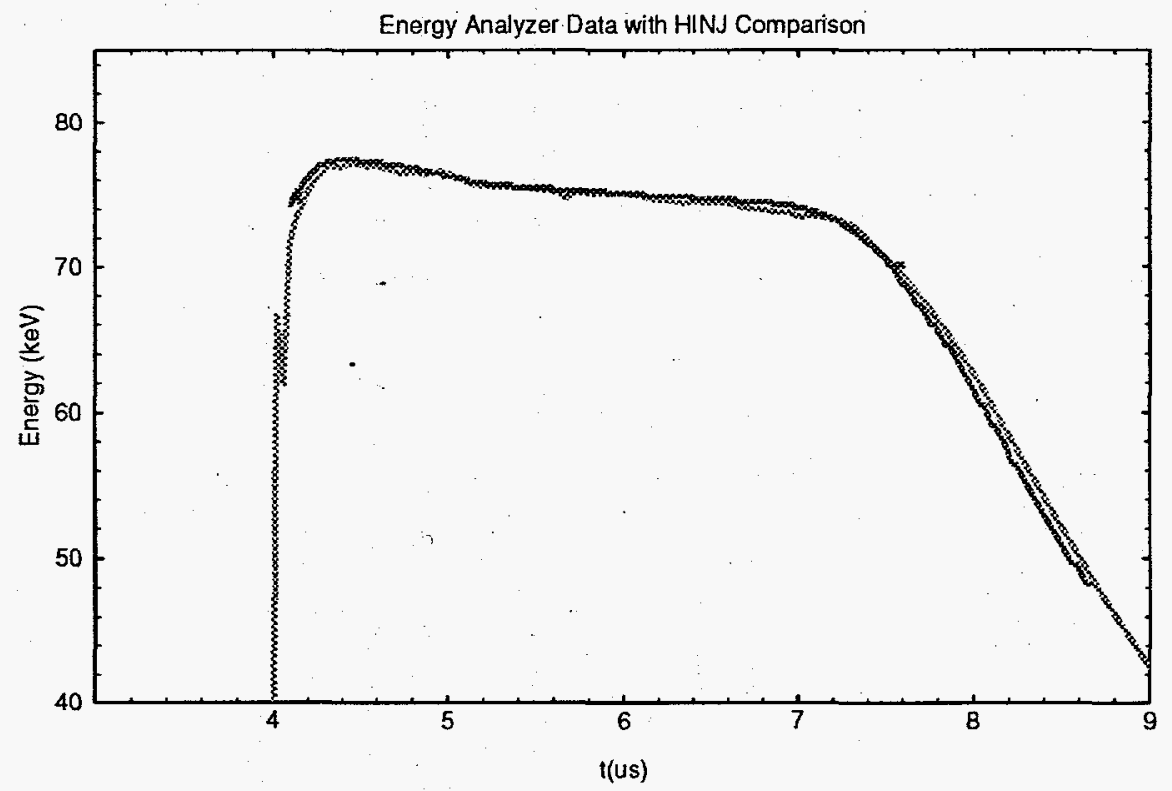

Figure 2. Peak energy signal (dark gray) and 1D simulations (light gray) vs. time at energy analyzer detector located $1.75 \mathrm{~m}$ downstream of the source. (The energy normalization and time zero point were adjusted for best fit of simulation to data).

Two-slit emittance scanner: In this dianostic, the beam impinges upon an aperture slit of width $0.1 \mathrm{~mm}$ (compared to a typical beam radius of $1 \mathrm{~cm}$ ), and $21 \mathrm{~cm}$ later passes through a second slit with the same dimension. These scanners may be placed at four different locations within the matching and magnetic transport sections. The transmitted beam current is measured. The position of both slits is varied and rms angles can be calculated. The 
results indicate a normalized emittance which grows from $0.025 \mathrm{~mm}$ mrad (just inside the matching section) to approximately $0.040 \mathrm{~mm} \mathrm{mrad}$, in about $4.7 \mathrm{~m}$ (at the end of of the magnetic transport section). (This compares to a normalized emittance at the beam source estimated to be approximately $0.020 \mathrm{~mm} \mathrm{mrad}$, based on its temperature and radius). The origin of this emittance growth is unknown, but our current suspicion is that mismatch oscillations are providing a source for beam thermalization. Although the emittance growth needs explanation, there is still an expectation of meeting our design goal of $0.10 \mathrm{~mm}$ mrad after insertion into the recirculator. Work on better beam matches that should alleviate much of this growth will be carried out after the new pulser is installed.

Capacitive probe: Capacitive probes (C-probes) are placed within the bores of the permanent magnet quadrupoles for a non-interceptive measurement of the beam centroid. These C-probes consist of cylindrical insulators with interiors that are copper plated on four equal sectors which run parallel to the longitudinal axis. The voltage on each segment can be measured, and the difference in voltage between the sum of the two left segments and the sum of the two right segments (for example) gives a measure which is nearly proportional to the offset of the beam centroid in the horizontal direction. A similar combination of voltages yields the position in the vertical direction. Preliminary measurements indicate that signals obtained from the probe in the presence of the beam are easily measured and have acceptable signal to noise ratio. It is anticipated that beam centroid position can be measured to $0.1 \mathrm{~mm}$ with these C-probes.

Rotating wire scanner: Another method of determining beam position and, in addition, of measuring the cross sectional profile of the beam has been explored using a rotating wire scanner. In this method, a $1 \mathrm{~mm}$ diameter wire intercepts a small portion of the beam, and either the current created by the ion striking the wire (and associated ejected electrons) is measured directly, or the electrons which are ejected from the wire by the beam impact are collected and measured. As an initial setup we have adapted a commercially available scanner (a National Electrostatics Corp. Model BPM82) which is shaped as a 45 degree helix. The helix can rotate at a rate on the order of one $\mathrm{Hz}$ and the beam is injected when the helix reaches a preset phase. The phase can then be varied, and thus a sequence of projections of the beam profile can be formed. (The advantage of using a helix is that with one wire two orthogonal directions can be measured.) These will provide checks for the $\mathrm{C}$-probes and can be parked outside the beam in normal operation. Initial results with this device have shown promise for direct and reproducible profile and centroid measurements and are being used as a check on the calibration of the C-probes. Position uncertainties of $\sim 0.1 \mathrm{~mm}$ are expected in these measurements. Slit scanner measurements downstream of the rotating wire scanner have shown negligible changes in the emittance. (In fact, some measurements show a small decrease in the emittance). Hence, these probes are being considered as "minimally" intercepting diagnostic for use in the main recirculating ring.

Gated beam imager: A final diagnostic under development is a gated beam imaging system. This system consists of an optical, time-gated CCD camera, together with a pepper-pot mask and a scintillating screen for the purpose of obtaining rapid measurements of the complete four dimensional phase space of the beam. As in the case of the slit scanner, after the beam 
passes through the array of circular apertures on the pepperpot mask, it expands, and the intensity, ellipticity and size of each miniature ellipse on the scintillating screen gives information about the detailed transverse phase space distribution function of the beam. Present experiments are examining saturation properties of the scintillating screen to establish the reliability and reproducibility of these measurements.

Spurious electron effects: Free electrons within the accelerator can be produced by stripping of the residual gas by the ion beam or the impact of stray beam particles against the structure, for example. In the electric focusing section, the electric fields of the electrodes readily separate the electrons from the ions, so that an accumulation of electrons in the potential well of the ions is unlikely to occur. In the magnetic transport section electrons could accumulate in the potential well of the ion beam between focusing magnets. (Within the quadrupoles, the small Larmor radius of the electrons would presumably prevent them from copropagating with the ions). In a previous study of space-charge dominated ion beams ${ }^{8}$, evidence for beam neutralization was presented at long pulse durations $(\sim 100 \mu \mathrm{s})$. By varying both the bias on the capacitive probes and the residual gas density, evidence for electron effects has been found in our experiment as well. At densities above about $\sim 10^{-6}$ torr there is an apparent decrease in the beam current over the duration of the pulse, particularly when the bias voltage on the C-probe exceeds the beam potential. This is indicative of electrons being collected during the pulse. This effect increases as the residual gas densities increase. At $\left(\sim 10^{-7}\right.$ torr (ten times the nominal operating residual gas pressure) these effects are minimal and would have minimal impact on beam propagation. We are continuing to refine these experiments to assess the effects of these stray electrons. Near term experiments are expected to have a more ideal injector waveform that will minimize overtaking and thereby minimize particle loss due to a mismatched beam head. This will enable a more concrete assessment of electron production due to particle stripping and the accompanying effects.

\section{Conclusion}

Previous studies have suggested that recirculating induction accelerators provide a potential lower cost approach for a heavy-ion fusion driver ${ }^{1}$. The LLNL experiments provide a small-scale test of most of the beam dynamics issues and many of the technology issues of high repetition rate induction acceleration relevant to a heavy ion driver for Inertial Fusion Energy. This experiment is being assembled as a series of experiments that will lead to a fully functioning recirculator. The current set of matching section and magnetic transport experiments have characterized the beam and tested a variety of probes which will be used when the complete ring is assembled. The physics of stray electrons is still being assessed, although they appear to play no significant role at the residual gas densities planned for the completed ring. We have briefly summarized the set of Heavy Ion Fusion beam experiments being carried out at LLNL. A more complete description is given in ref. 3 . 


\section{Acknowledgements}

This work was performed under the auspices of the U.S. D.O.E. by LLNL under contract no. W-7405-ENG-48 and at LBNL under contract no. DEAC03-76SF00098.

\section{References}

1. J.J. Barnard, A.L. Brooks, F. Coffield, F. Deadrick, L.V. Griffith, H.C. Kirbie, V.K. Neil, M.A. Newton, A.C. Paul, L.L. Reginato, W.M. Sharp, J. Wilson, S.S. Yu, and D.L. Judd, "Study of Recirculating Induction Accelerators as Drivers for Heavy Ion Fusion," Lawrence Livermore National Laboratory Report UCRL-LR-108095.

2. J.J. Barnard, F. Deadrick, A. Friedman, D.P. Grote, L.V. Griffith, H.C. Kirbie, V.K. Neil, M.A. Newton, A.C. Paul, W.M. Sharp, H.D. Shay, R.O. Bangerter, A. Faltens, C.G. Fong, D.L. Judd, E.P. Lee, L.L. Reginato, S.S. $\mathrm{Yu}$, and T.F. Godlove, "Recirculating Induction Accelerators as Drivers for Heavy Ion Fusion," Physics of Fluids B: Plasma Physics, 5, 2698 (1993).

3. T.J. Fessenden, J.J. Barnard, M.D. Cable, F.J. Deadrick, M.B. Neslon, T.C. Sangster, S. Eylon,H.S. Hopkins, "Intense Heavy-Ion Beam Transport with Electric and Magnetic Quadrupoles," submitted to Fusion Engineering and Design (1995).

4.R.O. Bangerter, "The Induction Approach to Heavy-Ion Inertial Fusion: Accelerator and Target Considerations," Il Nuovo Cimento, 106A, 1445, (1993).

5. A. Friedman, J.J. Barnard, M.D. Cable, D.A. Callahan, F.J. Deadrick, S. Eylon, T.J. Fessenden, D.P. Grote, H.A. Hopkins, V.P. Karpenko, D.L. Judd, H.C. Kirbie, D.B. Longinotti, S.M. Lund, L.A. Nattrass, M.B. Neslon, M.A. Newton, W.M. Sharp, and S.S. Yu, "Recirculating Induction Accelerators for Inertial Fusion: Prospects and Status," submitted to Fusion Engineering and Design (1995).

6. J.J. Barnard, M.D. Cable, D.A. Callahan, T.J. Fessenden, A. Friedman, D.P. Grote, D.L. Judd, S.M. Lund, M.A. Newton, W.M. Sharp, and S.S. Yu, "Physics Design and Scaling of Recirculation Induction Accelerators: From Benchtop Prototypes to Drivers," submitted to Fusion Engineering and Design (1995).

7. A. Friedman, "Induction-Accelerator Heavy Ion Fusion: Status and Beam Physics Issues," these proceedings.

8. J. Klabunde, M. Reiser, A. Schonlein, P. Spadtke, J. Struckmeier, "Studies of Heavy Ion Beam Transport in a Magnetic Quadrupole Channel," IEEE Transactions on Nuclear Science, Vol. NS-30, 2543 (1983).

9. M.G. Tiefenback, "Space-Charge Limits on the Transport of Ion Beams in a Long Alternating Gradient System," Ph. D. Dissertation, University of California, Berkeley, LBL-22465, HIF AN-352.

10. M.A. Newton, F.J. Deadrick, R.L.Hanks, H.C. Kirbie, V.P. Karpenko, L.A. Nattrass, "Engineering Developments for a Small-Scale Recirculator," submitted to Fusion Engineering and Design (1995). 
11. M. Lampel and M. Tiefenback, "An Applied Voltage to Eliminate Current Transients in a One-Dimensional Diode," App. Phys. Lett. 43, 57, (1983). 